Since the Hamiltonian groups of order $2^{m}$ constitute such a well known and important category of groups it may be of interest to note here that it results from the preceding developments that these groups are characterized by the facts that they admit three-fourths automorphisms and that the continued product of these three automorphisms is the identity. This category is also characterized by the fact that it is composed of the groups whose three three-fourths automorphisms are inner automorphisms of order 2. The entire category of Hamiltonian groups may be similarly characterized by the fact that it is composed of the groups whose centrals involve no operators of order 4 and which admit such three three-fourths automorphisms that in their continued product all the operators of the central correspond to their inverses and at least one operator in each central co-set corresponds to itself.

The University of Illinois

\title{
THE ROLE OF DIRECTION IN THE VARIATIONS OF A DEFINITE INTEGRAL IN SPACE
}

\section{BY LOUIS BRAND}

1. Introduction. In the space problem of the calculus of variations, whether treated in the parametric form as by Bliss and Mason, $\dagger$ or in terms of angle as by Rider $\ddagger$ or Sakellariou, $\$$ the Euler equations are three in number but connected by a relation. Thus but two of the equations are independent. The object of this paper is to show that two independent Euler equations arise from variations parallel

* Presented to the Society, April 16, 1927.

$\dagger$ Mason, M. and Bliss, G. A., The properties of curves in space which minimize a definite integral, Transactions of this Society, vol. 9 (1908), pp. 440-466.

$\ddagger$ Rider, P. R., The space problem of the calculus of variations in terms of angle, American Journal of Mathematics, vol. 39 (1917), pp. 241-256.

$\S$ Sakellariou, N., The space problem of the calculus of variations, Palermo Rendiconti, vol. 44 (1920), pp. 53-68. 
to principal normal and binormal, respectively, of the curve giving an extremum. Tangential variations yield nothing but an identity in partial differentiation. The two independent Euler equations may be replaced by a vector equation which involves a certain vector $Q$. This vector plays an important part in the entire theory covering the first and second variations of the integral.

2. The Euler Equations. Consider the integral

$$
I=\int_{0}^{l} F(\boldsymbol{r}, \boldsymbol{t}) d s
$$

taken along a curve $\Gamma$ in space joining two fixed points. Here $F$ is a scalar point function of the position vector $\boldsymbol{r}$ and its derivative $d r / d s=t$ (the unit tangent vector) which has continuous partial gradients of the second order with respect to $\boldsymbol{r}$ and $t$. We note in passing that this formulation of the problem applies equally well to variations in space or on a given surface.

Now let the curve $\Gamma, \boldsymbol{r}=\boldsymbol{r}(s)$, give $I$ an extreme value. We replace $\Gamma$ by another curve $\Gamma_{1}$ :

$$
\boldsymbol{r}_{1}=\boldsymbol{r}+\epsilon(u t+v p+w b)
$$

between the given points, where $u, v, w$ are three independent functions of $s$ of class $C^{\prime}$ which vanish for $s=0$ and $s=l$, and $t, p, b$ are unit vectors in the direction of the tangent, principal normal and binormal along $\Gamma$, chosen so that $t p b$ form a right-handed set. Thus (1) gives explicit representation to variations in the directions of $t, p$ and $b$.

The value of $I$ along $\Gamma_{1}$ is

where

$$
I(\epsilon)=\int_{0}^{l} F\left(\boldsymbol{r}_{1}, \boldsymbol{t}_{1}\right) \phi(s, \epsilon) d s,
$$

$$
\phi(s, \epsilon)=d s_{1} / d s,
$$

and if $\nabla_{r} F, \nabla_{t} F$ denote partial gradients of $F$ with respect to $\boldsymbol{r}$ and $\boldsymbol{t}$, 
(2) $I^{\prime}(\epsilon)=\int_{0}^{l}\left\{\left[\frac{\partial r_{1}}{\partial \epsilon} \cdot \nabla_{r_{1}} F+\frac{\partial t_{1}}{\partial \epsilon} \cdot \nabla_{t_{1}} F\right] \phi+F \frac{\partial \phi}{\partial \epsilon}\right\} d s$.

On differentiating (1) with respect to $\epsilon$ and with respect to $s$ and making use of Frenet's formulas:

$$
\frac{d t}{d s}=\kappa p, \quad \frac{d p}{d s}=-\kappa t+\tau b, \quad \frac{d b}{d s}=-\tau p,
$$

we obtain

$$
\begin{gathered}
\frac{\partial \boldsymbol{r}_{1}}{\partial \epsilon}=u t+v p+w b \\
\text { (4) } \quad t_{1} \phi(s, \epsilon)=\left(1+\epsilon u^{\prime}-\epsilon \kappa v\right) t+\epsilon\left(\kappa u+v^{\prime}-\tau w\right) p \\
+\epsilon\left(\tau v+w^{\prime}\right) b
\end{gathered}
$$

where the primes denote differentiation with respect to $s$. We now differentiate (4) with respect to $\epsilon$ :

whence

$$
\begin{aligned}
\frac{\partial t_{1}}{\partial \epsilon} \phi+t_{1} \frac{\partial \phi}{\partial \epsilon}=\left(u^{\prime}-\kappa v\right) t & +\left(\kappa u+v^{\prime}-\tau w\right) p \\
& +\left(\tau v+w^{\prime}\right) b
\end{aligned}
$$

$$
\begin{gathered}
\frac{\partial \phi}{\partial \epsilon}=\left(u^{\prime}-\kappa v\right) t \cdot t_{1}+\left(\kappa u+v^{\prime}-\tau w\right) p \cdot t_{1} \\
+\left(\tau v+w^{\prime}\right) b \cdot t_{1} .
\end{gathered}
$$

Putting $\epsilon=0$ in (4), (6) and (5) in turn now gives

$$
\begin{gathered}
\phi(s, 0)=1, \quad \phi_{\epsilon}(s, 0)=u^{\prime}-\kappa v, \\
\left.\frac{\partial t_{1}}{\partial \epsilon}\right|_{\epsilon=0}=\left(\kappa u+v^{\prime}-\tau w\right) p+\left(\tau v+w^{\prime}\right) b \\
=(u t)^{\prime}+(v p)^{\prime}+(w b)^{\prime}+\left(\kappa v-u^{\prime}\right) t .
\end{gathered}
$$

Finally we note that for a given $\boldsymbol{r}, \boldsymbol{t}$ can only vary over a unit sphere; hence $t \cdot \nabla_{t} F=0$. With these results and (3), (2) becomes

$$
\begin{gathered}
I^{\prime}(0)=\int_{0}^{l}\left\{(u t+v p+w b) \cdot \nabla_{r} F+\left[(u t)^{\prime}+(v p)^{\prime}\right.\right. \\
\left.\left.+(w b)^{\prime}\right] \cdot \nabla_{t} F+\left(u^{\prime}-\kappa v\right) F\right\} d s
\end{gathered}
$$


and by the usual integration by parts

$$
\begin{aligned}
& I^{\prime}(0)=\int_{0}^{l}\left\{u\left(t \cdot \nabla_{r} F-t \cdot \frac{d}{d s} \nabla_{t} F-\frac{d F}{d s}\right)\right. \\
& \left.\quad+v\left(p \cdot \nabla_{r} F-p \cdot \frac{d}{d s} \nabla_{t} F-\kappa F\right)+w\left(b \cdot \nabla_{r} F-b \cdot \frac{d}{d s} \nabla_{t} F\right)\right\} d s .
\end{aligned}
$$

Putting $v=w=0, w=u=0, u=v=0$ in turn, the condition $I^{\prime}(0)=0$ yields the three Euler equations:

$$
\begin{array}{r}
t \cdot \nabla_{r} F-t \cdot \frac{d}{d s} \nabla_{t} F-\frac{d F}{d s}=0, \\
p \cdot \nabla_{r} F-p \cdot \frac{d}{d s} \nabla_{t} F-\kappa F=0, \\
b \cdot \nabla_{r} F-b \cdot \frac{d}{d s} \nabla_{t} F=0 .
\end{array}
$$

3. The Euler Equation for the Tangential Variation is an Identity. To show this we differentiate the relation $t \cdot \nabla_{t} F=0$ with respect to $s$ :

$$
\frac{d t}{d s} \cdot \nabla_{t} F+t \cdot \frac{d}{d s} \nabla_{t} F=0 .
$$

Thus (7) reduces to an identity in partial differentiation,

$$
\frac{d F}{d s}=\frac{d \boldsymbol{t}}{d s} \cdot \nabla_{r} F+\frac{d \boldsymbol{t}}{d s} \cdot \nabla_{t} F .
$$

The two independent Euler equations thus arise from variations parallel to the principal normal and binormal.

4. The Euler Equation in Vector Form. Equations (7), (8), (9) are the $t, p$ and $b$ components of the vector equation

$$
\nabla_{r} F-\frac{d \boldsymbol{Q}}{d s}=0, \text { where } \boldsymbol{Q}=F t+\Delta_{t} F .
$$

This equation puts in evidence three cases of immediate integrability, namely when $\nabla_{r} F$ is zero, parallel to a fixed vector $\boldsymbol{k}$, or perpendicular to $\boldsymbol{k}$; in these cases $\boldsymbol{Q}, \boldsymbol{k} \times \boldsymbol{Q}$ 
or $\boldsymbol{k} \cdot \boldsymbol{Q}$ are respectively constant. Equation (10) is of the second order when $\nabla_{t} Q \neq 0$; it degenerates when $Q$ is a function of $\boldsymbol{r}$ alone, that is, only when $F$ has the form $t \cdot f(\boldsymbol{r})$.

In a paper presented to the Society, $\dagger$ I have shown that (10) follows directly from the condition $I^{\prime}(0)=0$ by using variations of an arbitrary but fixed direction, $\boldsymbol{r}_{1}=\boldsymbol{r}+\boldsymbol{\epsilon} \boldsymbol{c}$, where $c$ is a constant vector. The second variation then takes the form $I^{\prime \prime}(0)=c \cdot \Phi \cdot c$, where $\Phi$ is a tensor of the second order.

To show the part played by the vector $Q$ in the general theory we merely state the following results. The Weierstrass-Erdmann corner condition is

$$
Q\left(r, t^{+}\right)=Q\left(r, t^{-}\right) .
$$

In a regular problem the tensor $\nabla_{t} Q$, which is planar and symmetric, is not zero; its second scalar invariant (in the sense of Gibbs) must be positive or zero in the case of a minimum. This is the analogue of the Legendre condition.

If $t=t^{*}$ in a field of extremals and $Q^{*}=Q\left(r, t^{*}\right)$, the Euler equation gives

$$
t^{*} \times \operatorname{rot} Q^{*}=0
$$

in this field. The Hilbert invariant integral is

$$
I^{*}=\int Q^{*} \cdot t d s .
$$

From this we have the fundamental theorem of Weierstrass

$$
\nabla I=\int\left(Q-Q^{*}\right) \cdot t d s
$$

The integrand $\left(Q-Q^{*}\right) \cdot t$ is the $E$-function. The transversality condition is simply $Q^{*} \cdot t=0$.

In the corresponding problem on a surface of unit normal $\boldsymbol{n}$, the Euler equation is

$\dagger$ December 1, 1928, The calculus of variations from a vector standpoint. See this Bulletin, vol., 35 (1929), p. 153. 


$$
n \times\left(\nabla_{r} F-\frac{d Q}{d s}\right)=0
$$

when $Q$ is defined as above. The tensor $\nabla_{t} Q$ is now symmetric and linear and its first scalar invariant must be positive or zero in the case of a minimum. In a field of extremals

$$
n \cdot \operatorname{rot} Q^{*}=0 \text {. }
$$

The Hilbert integral, the $E$-function and the transversality condition are the same as before. $\dagger$ The last equation and $Q^{*} \cdot n=0$ show that $Q^{*}$ is the surface gradient of a scalar function $W$. The curves $W=$ const. are the transversals of the field.

We note, finally, that analogous results hold in the problem of finding an extremum for a surface integral

$$
I=\int F(\boldsymbol{r}, n) d \sigma
$$

bounded by a fixed closed curve. The Euler equation in this case is

$$
n \cdot \nabla_{r} F+\operatorname{div} P=0 \text { where } P=F n+\nabla_{n} F
$$

and div denotes a surface divergence. That $P$ has the same general form as $Q$ is apparent. If $n=n^{*}$ in a field of extremal surfaces and $P^{*}=P\left(r, n^{*}\right)$, then

$$
\operatorname{div} P^{*}=0 \text {. }
$$

The Hilbert integral, the $E$-function and the transversality condition are respectively

$$
\int P^{*} \cdot n d \sigma, \quad\left(P-P^{*}\right) \cdot n, \quad P^{*} \cdot n=0 .
$$

These results will be developed elsewhere.

The University of Cincinnati

$\dagger$ From this fact we have an immediate proof of a theorem given by Douglas in this Bulletin (vol. 32 (1926), pp. 669-674) to the effect that transversality of an integral relative to a surface $\Sigma$ is the section by $\Sigma$ of its space transversality. 